\title{
Financement des soins en prison
}

Madame, Monsieur,

La Fédération des médecins suisses (FMH) et la Conférence des médecins pénitentiaires suisses (CMPS) sont préoccupées par la qualité des soins en prison, car l'accès aux soins est toujours plus souvent lié à une participation financière des personnes détenues.

En février 2019, la Commission centrale d'éthique de l'ASSM publiait une déclaration soulignant l'importance d'une prise en charge médicale efficace et de qualité équivalente à celle de la population générale, et garantie indépendamment du statut de résidence [1].

Les directives médico-éthiques sur l'exercice de la médecine auprès des personnes détenues stipulent que la prise en charge médicale doit être accessible et gratuite. Une participation adéquate aux frais n'est exigible qu'à titre exceptionnel.

De nombreux médecins exerçant en milieu carcéral constatent que l'accès aux soins y devient toujours plus compliqué. Ils passent ainsi beaucoup plus de temps et d'énergie en demandes de prise en charge et en négociations avec les autorités d'exécution des peines et les services sociaux pour permettre à leurs patients d'accéder aux soins.

Récemment, les autorités d'exécution des peines d'un canton ont décidé que les factures de médecin ne devaient plus leur être envoyées à elles, mais directement au patient détenu. Ce n'est qu'après trois rappels non payés et documentés que le médecin peut s'adresser aux autorités pénitentiaires pour paiement.

D’autres cantons ont commencé cette année à facturer les prestations de soins à la personne détenue, indépendamment de son statut d'assuré ou de sa situation financière, généralement précaire. Pour rappel, le revenu moyen d'une personne détenue est inférieur à 500 francs par mois, pour autant qu'elle ait accès à un travail rémunéré

La médecine pénitentiaire ne peut ainsi plus remplir certaines fonctions, pourtant essentielles en termes de santé publique, et sa qualité est en péril. Comment un médecin peut-il détecter des maladies infectieuses telles que la tuberculose, le VIH ou l'hépatite C si son patient ne vient plus consulter ou si, comme souvent observé entre-temps, il ne se soumet plus aux examens et traitements prévus, par crainte d'une participation financière?

Les réglementations des autorités d'exécution des peines concernant le financement des soins en prison incitent certains détenus à ne pas se faire soigner. Une fonction essentielle de santé publique ne pourra ainsi plus être remplie, à savoir détecter précocement et traiter les maladies, de sorte que la personne ne présente pas de risque sanitaire pour la société, y compris après sa sortie de prison.

Les prestations médicales en prison doivent être équivalentes à celles dont bénéficie la population générale. Peu importe si un détenu est soumis ou non à la LAMal: les prestations ne doivent pas être inférieures au catalogue des prestations de base. Par ailleurs, les cantons appliquent une diversité de systèmes pour la prise en charge des coûts, ce qui entraîne certaines inégalités de traitement. Il conviendrait d'élaborer une solution nationale uniforme, et de faire en sorte qu'à l'avenir, toutes les personnes détenues soient soumises à l'assurance-maladie obligatoire.

Veuillez agréer, Madame, Monsieur, nos salutations les meilleures.

Prof. Hans Wolff, président CMPS

Dr Jürg Schlup, président FMH

\section{Références}

1 Prise de position de la Commission centrale d'éthique de l'ASSM «Financement des prestations médicales en milieu carcéral», https://www.samw.ch/fr/Publications/Prises-de-position.html

2 Rapport de la CNPT sur la prise en charge médicale dans les établissements de privation de liberté en Suisse, https://www.nkvf.admin.ch/nkvf/fr/home/publikationen/ newsarchiv/2019/2019-11-14.html 